\title{
Congenital unilateral hypoplasia of depressor anguli oris muscle in adult
}

\section{Suk Joon Oh}

Department of Burn Reconstructive Surgery, Bestian Seoul Hospital, Seoul, Korea

This article contains Supplemental Video S1.

\begin{abstract}
Congenital hypoplasia of the depressor anguli oris muscle is a rare cause of asymmetrical crying facies in newborns. The clinical manifestations range from mild to severe asymmetry and may persist up to adulthood. In the current case, the patient did not exhibit other congenital anomalies or paralysis of other branches of the facial nerve. This adult patient presented with severe asymmetrical lower lip deformity during full mouth opening since birth. A chromosomal study for the detection of 22q gene deletion yielded negative results. The electromyography findings of the lower lip were insignificant. Depressor labii inferioris muscle resection was not effective, but bidirectional (horizontal and vertical) fascia lata grafting improved the aesthetic appearance of the asymmetrical lower lip. The patient showed improved lower lip symmetry during full mouth opening at 1 year after the surgery. Therefore, the details of this rare case are reported herein.
\end{abstract}

Keywords: Congenital / Fascia lata / Partial unilateral facial paralysis / Reconstruction

\section{INTRODUCTION}

Congenital hypoplasia of the depressor anguli oris (DAO) muscle is a rare cause of asymmetrical crying facies (ACF) in newborns. Parmelee [1] first described facial weakness apparent during crying. Cayler [2] reported the cases of five patients with both congenital partial lower facial paresis involving unilateral lower lip depressors and heart defects.

Neonatal ACF is estimated to occur in $0.6 \%$ of infants. The clinical presentation of children with ACF is characterized by drooping of the lower lip on the intact side during crying or mouth opening. The cause is either facial nerve compression or defective facial muscle and/or nerve development. Spontaneous recovery is expected with the former but not necessarily with the latter etiology [3].

The diagnosis can be established by the clinical features and/

Correspondence: Suk Joon Oh

Department of Burn Reconstructive Surgery, Bestian Seoul Hospital, 429 Dogok-

ro, Gangnam-gu, Seoul 06208, Korea

E-mail: sjoh46@nate.com

Received May 27, 2019 / Revised July 22, 2019 / Accepted August 7, 2019 or electromyography (EMG) [4]. Children with congenital unilateral hypoplasia of the DAO muscle may also exhibit a wide variety of anomalies involving the cardiovascular, gastrointestinal, genitourinary, skeletal, central nervous systems, and additional anomalies.

Although several dynamic and static reconstruction methods have been reported, passive surgical interventions have been introduced to weaken unaffected aspects through techniques such as selective surgical mandibular neurectomy and myomectomy. However, these methods tend to result in a lack of motion during the facial expression of emotions [5].

In the current case, symmetry of the lower lip during full mouth opening was restored after bidirectional fascia lata grafting.

\section{CASE REPORT}

A 38-year-old male patient presented with severe asymmetrical lower lip deformity during full mouth opening since birth (Fig. 1A). He presented mild asymmetry of corners during mouth 
closing (Fig. 1B). This patient did not exhibit other congenital anomalies or paralysis of other branches of the facial nerve. $\mathrm{He}$ had not undergone any previous surgeries for congenital lower lip palsy. His mother showed mild lower lip asymmetry. A chromosomal study for the detection of $22 \mathrm{q}$ gene deletion yielded negative results. The EMG findings of the lower lip were insignificant.

Depressor labii inferioris (DLI) muscle resection was performed on the right (normal) side under local anesthesia. However, the asymmetrical lower lip deformity was not improved at 6 months after the first operation.

This patient was suspected of congenital hypoplasia of the DAO muscle. A bidirectional (horizontal and vertical) fascia lata strip $(15 \times 1 \mathrm{~cm})($ Fig. 2) was harvested from the right thigh using a fascia lata stripper and grafted through the vermillionmucosal junction and submandibular incisions under local anesthesia. A horizontal fascia lata graft $(5 \times 1 \mathrm{~cm}$ in size $)$ was fixed at three points on the anterior aspect of the orbicularis oris muscle from the mid-portion of the right side of the lower lip to the left modiolus. The upper, looped end of the doubled vertical fascia lata graft strip $(5 \times 2 \times 1 \mathrm{~cm})$ was fixed on the mid-portion of the horizontal fascial graft in the cranial direction, after which it was overturned and passed behind the orbi-
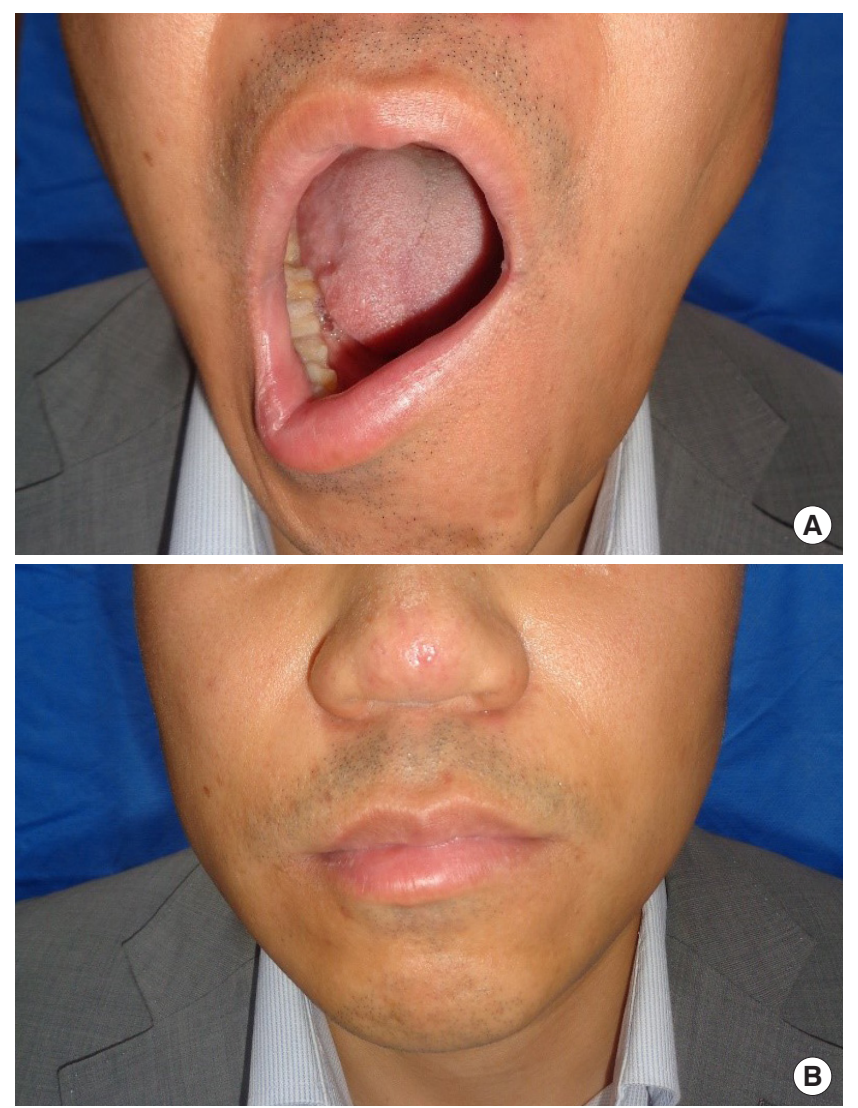

Fig. 1. (A, B) Preoperative appearance of opened and closed mouth. cularis oris muscle. The other end of the fascial graft was sutured to the mandibular periosteum through submandibular incisions (Fig. 3).

Early postoperative undercorrection of the asymmetrical lip required correction of the vertical strips. However, significant edema occurred at the dissected part of the lower lip, and correction was delayed. The vertical strip on the mandibular edge was detached and refixed to the periosteum of the mandibular edge at a position providing more correction six months later.

The patient's lip was more symmetrical with the mouth either opened (Fig. 4A) or closed (Fig. 4B) at 1 year after the correction surgery (Supplemental Video S1). The operative scar is inconspicuous, and his left thigh shows minimal donor-site morbidity.

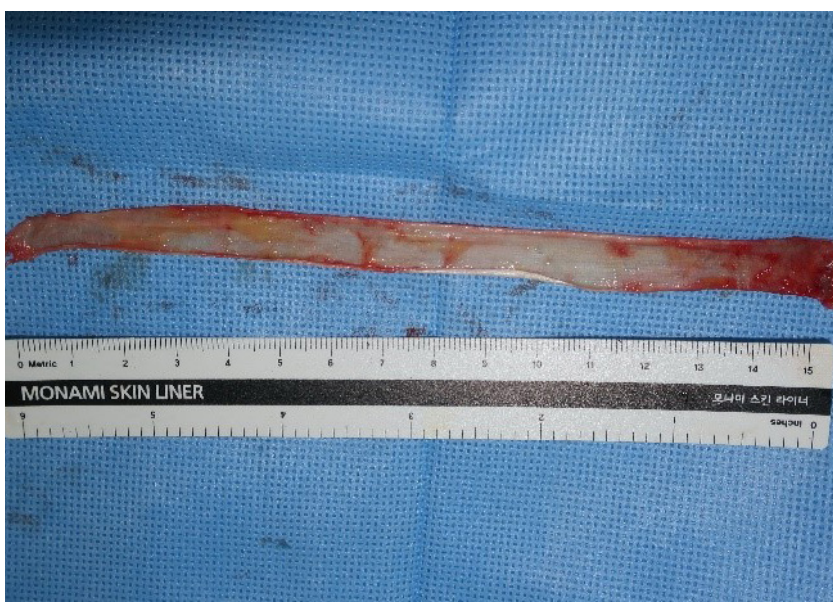

Fig. 2. Harvested fascia lata strip $15 \times 1 \mathrm{~cm}$ in size.

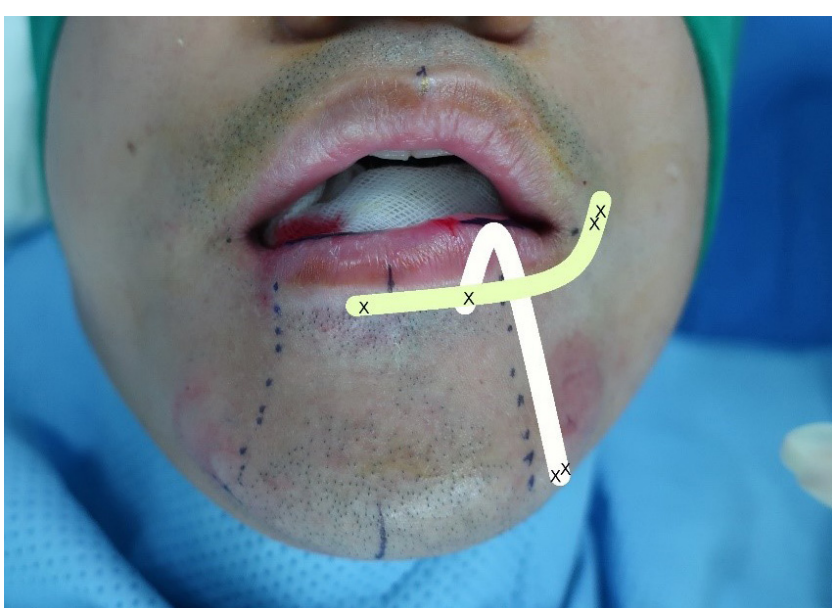

Fig. 3. Schematic of this procedure. A horizontal fascia strip grafted through intraoral incisions corrects dislocation of the center of the lower lip, and a vertical fascia strip grafted between the horizontal fascia and the mandibular border draws the affected lip down through simultaneous movement of the lower lip with the mandible (X marks fixed sutures). 

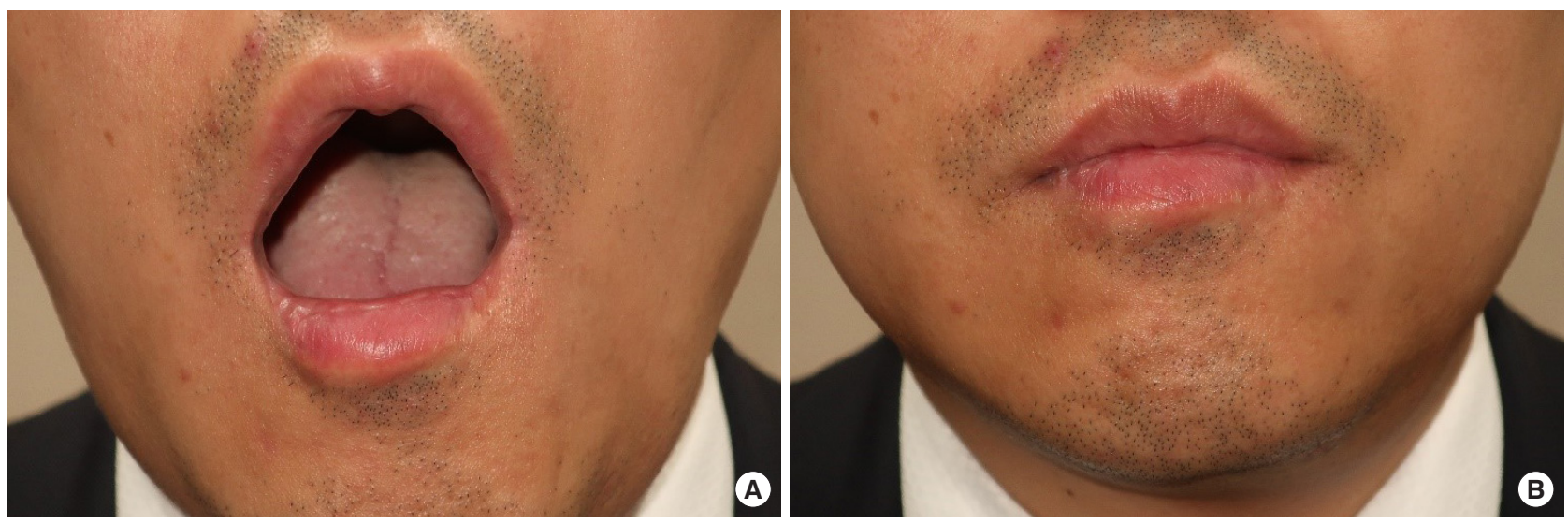

Fig. 4. (A, B) Postoperative appearance of opened and closed mouth 1 year after surgery.

\section{DISCUSSION}

Congenital hypoplasia of the DAO muscle, known as neonatal $\mathrm{ACF}$, is characterized by facial asymmetry, which usually occurs when the infant is crying because the half-normal lower lip manifests an appearance of being pulled down and outward [6]. Various instances of ACF associated with congenital anomalies have been reported. Cayler reported five cases [2] and 30 cases [7] of neonatal ACF associated with congenital heart defects, termed craniofacial syndrome.

The incidence of hypoplasia of the DAO muscle in newborn infants' ranges from $0.26 \%$ [8] to $0.82 \%$ [4]. A total of 24,472 newborns were evaluated in prospective studies, and 149 infants $(0.6 \%)$ were found to have neonatal ACF [3].

Pape and Pickering [6] were the first to recommend that although hypoplasia of the DAO muscle could be an isolated finding, its presence should lead to a search for other anomalies. The reported frequency of major malformations in infants with neonatal ACF ranges widely from 5\% to 77\%. Approximately $10 \%$ of developmental cases have associated major malformations [3]. Regarding the sex distribution, the male-to-female ratio was reported to be 2:1 by Lin et al. [9]. However, other case series have reported similar data $[4,10]$.

As in our case, left-sided predominance has been reported in several case series $[1,4,8,10,11]$, while right-side predominance has been reported in other series $[6,12]$.

Pasick et al. [13] reported an incidence of 22q11.2 deletion of $14 \%$ in 836 patients, which is significantly higher than the incidence in the general population. Therefore, they suggested that newborns with ACF should be referred for further screening for $22 \mathrm{q} 11.2$ deletion syndrome. They advised searching for a 22 q11.2 microdeletion in only those with cardiofacial syndrome or both neonatal ACF and additional anomalies. A chromosomal study for the detection of $22 \mathrm{q}$ gene deletion yielded negative results in the present case.

The pathogenesis of hypoplasia of the DAO muscle is unknown [10]. Earlier studies have suggested that intrauterine molding or subclinical viral infections may be the cause of this defect. More than half of 37 patients had one or more similarly affected relatives in consecutive generations in three families $[4,14]$ suggesting an autosomal-dominant type of inheritance with variable expressivity. The mother of the present patient showed a mild form of left congenital hypoplasia of the DAO muscle.

An EMG study reported by Papadatos et al. [4] confirmed the myogenic nature by such findings as a paucity of diminution of the motor units of short duration and relatively low voltage in 10 out of 15 affected and examined patients. There was no correlation between the degree of lower lip asymmetry and the paucity of motor units. The conduction times and results of nerve excitability studies were normal and confirmed the intactness of the facial nerves.

EMG showed signs of hypoplasia of the DAO muscle in 41 of 51 infants with ACF. There was no question of total muscular agenesis in any case [15]. The EMG findings of the lower lip of the patient in the present case were insignificant.

Edgerton [16] first described transfer of the anterior belly of the digastric muscle. This involved dividing the mandibular insertion of the muscle and attaching it to the lower lip with a fascia lata graft. Terzis and Kalantarian [17] have also described the use of platysma muscle transfer. Yavuzer and Jackson [18] described a strategy consisting of excising a wedge of the lower lip from the non-paralyzed side and suspending the non-paralyzed orbicularis muscle through a nasolabial incision.

DLI muscle resection is a simple and effective procedure for the treatment of marginal mandibular nerve palsy. The results 
are permanent and predictable. Lower lip symmetry is produced both at rest and with facial animation, without causing a functional deficit [11]. For congenital lower lip palsy in children, a simple reconstruction procedure using bidirectional (horizontal and vertical) fascial grafts has been previously reported [5]. This technique is considered to be a well-balanced surgical intervention regarding its simplicity, minimal invasiveness and resulting parent [5] and adult patient [19] satisfaction.

In summary, this report describes a case of congenital unilateral hypoplasia of the DAO muscle in an adult. Although this is a single isolated case, bidirectional (horizontal and vertical) fascial grafts could also be effective for adult patients with congenital unilateral hypoplasia of the DAO muscle. This method is easier in adults than in children because revision surgery can be easily performed, and little consideration of postoperative facial growth is needed. In adult patients, surgery can be performed under local anesthesia. However, the problem of determining the appropriate tension of the vertical strip under general anesthesia must be resolved.

\section{NOTES}

\section{Conflict of interest}

No potential conflict of interest relevant to this article was reported.

\section{Ethical approval}

The study was approved by the Institutional Review Board of Bestian Seoul Hospital (IRB No. 2019-01-001) and performed in accordance with the principles of the Declaration of Helsinki. Written informed consent was obtained.

\section{Patient consent}

The patient provided written informed consent for the publication and the use of his images.

\section{ORCID}

Suk Joon Oh https://orcid.org/0000-0001-7793-6198

\section{Supplementary material}

Supplemental Video S1. Mouth motion at 1 year after bidirectional fascia lata grafting.

Supplemental data can be found at:

https://doi.org/10.7181/acfs.2019.00297

\section{REFERENCES}

1. Parmelee Ah. Molding due to intra-uterine posture: facial pa- ralysis probably due to such molding. Am J Dis Child 1931;42:1155-9.

2. Cayler GG. An "epidemic" of congenital facial paresis and heart disease. Pediatrics 1967;40:666-8.

3. Sapin SO, Miller AA, Bass HN. Neonatal asymmetric crying facies: a new look at an old problem. Clin Pediatr (Phila) 2005; 44:109-19.

4. Papadatos C, Alexiou D, Nicolopoulos D, Mikropoulos H, Hadzigeorgiou E. Congenital hypoplasia of depressor anguli oris muscle. A genetically determined condition? Arch Dis Child 1974;49:927-31.

5. Udagawa A, Arikawa K, Shimizu S, Suzuki H, Matsumoto H, Yoshimoto $S$, et al. A simple reconstruction for congenital unilateral lower lip palsy. Plast Reconstr Surg 2007;120:238-44.

6. Pape KE, Pickering D. Asymmetric crying facies: an index of other congenital anomalies. J Pediatr 1972;81:21-30.

7. Cayler GG, Blumenfeld CM, Anderson RL. Further studies of patients with the cardiofacial syndrome. Chest 1971;60:161-5.

8. Levin SE, Silverman NH, Milner S. Hypoplasia or absence of the depressor anguli oris muscle and congenital abnormalities, with special reference to the cardiofacial syndrome. S Afr Med J 1982;61:227-31.

9. Lin DS, Huang FY, Lin SP, Chen MR, Kao HA, Hung HY, et al. Frequency of associated anomalies in congenital hypoplasia of depressor anguli oris muscle: a study of 50 patients. Am J Med Genet 1997;71:215-8.

10. Lahat E, Heyman E, Barkay A, Goldberg M. Asymmetric crying facies and associated congenital anomalies: prospective study and review of the literature. J Child Neurol 2000;15:80810.

11. Hussain G, Manktelow RT, Tomat LR. Depressor labii inferioris resection: an effective treatment for marginal mandibular nerve paralysis. Br J Plast Surg 2004;57:502-10.

12. Bawle EV, Conard J, Van Dyke DL, Czarnecki P, Driscoll DA. Seven new cases of Cayler cardiofacial syndrome with chromosome 22q11.2 deletion, including a familial case. Am J Med Genet 1998;79:406-10.

13. Pasick C, McDonald-McGinn DM, Simbolon C, Low D, Zackai E, Jackson O. Asymmetric crying facies in the 22q11.2 deletion syndrome: implications for future screening. Clin Pediatr (Phila) 2013;52:1144-8.

14. Silengo MC, Bell GL, Biagioli M, Guala A, Bianco R, Strandoni $\mathrm{P}$, et al. Asymmetric crying facies with microcephaly and mental retardation: an autosomal dominant syndrome with variable expressivity. Clin Genet 1986;30:481-4.

15. Renault F. Facial electromyography in newborn and young infants with congenital facial weakness. Dev Med Child Neurol 2001;43:421-7. 
16. Edgerton MT. Surgical correction of facial paralysis: a plea for better reconstructions. Ann Surg 1967;165:985-98.

17. Terzis JK, Kalantarian B. Microsurgical strategies in 74 patients for restoration of dynamic depressor muscle mechanism: a neglected target in facial reanimation. Plast Reconstr Surg 2000; 105:1917-31.
18. Yavuzer R, Jackson IT. Partial lip resection with orbicularis oris transposition for lower lip correction in unilateral facial paralysis. Plast Reconstr Surg 2001;108:1874-9.

19. Kubota Y, Kuroki T, Koizumi T, Udagawa A. Bidirectional fascia graft for congenital unilateral lower lip palsy in an adult. J Plast Reconstr Aesthet Surg 2009;62:e121-2. 